\title{
Neglected tropical crops?
}

\author{
Medical science has acknowledged that research resources are not always directed where they will be \\ most effective. Is it time that we paid similar attention to blind spots within the plant sciences?
}

Over the past decade or so, there has been an increased awareness of what are known as neglected tropical diseases (NTDs). These are a diverse group of communicable diseases that, despite affecting a vast number of the world's population and causing substantial economic costs for the countries where they are endemic, attract little money for research or treatment. In 2012 the World Health Organization published a roadmap for the eradication or control of 17 NTDs by the end of this decade. Their list includes dengue, leprosy, guinea worm, leishmaniasis, schistosomiasis, yaws and other micro- and macroparasitic conditions.

NTDs occur predominantly in tropical and subtropical countries. They are associated with poor sanitation and lifestyles that bring peoples into close contact with infectious vectors such as domestic animals and livestock. Put simply, they are diseases of poverty - one substantial aspect of which is a meagre and insecure food supply. Why, then, are crops that form a substantial part of the global diet not the subject of equally substantial research and development?

The Food and Agriculture Organization of the United Nations (FAO) collects annual statistics on global crop production (http://faostat3.fao.org/home/). The most recently available data is for 2013; it shows that wheat, maize and rice are the most widely grown crops, having global cultivated areas of 219, 185 and 165 million hectares, respectively. The yields from each crop vary, so that maize produces the largest harvest weight of the three at just over 1 billion tonnes for the year - but the impressive yields obtained from sugarcane make it the largest crop of all by harvest weight (1.9 billion tonnes), despite being only the tenth by cultivated area.

Assessing research investment is a harder task. No precise statistics exist, but a fair proxy would be numbers of published research papers. The exact numbers depend on the source (PubMed, Google Scholar, ISI, Scopus, and so on), but there is a clear correlation between the number of research articles and cultivated area for common crop species (Fig. 1). Wheat, rice and maize dominate the crop research field, but the roughly linear correlation can be used to identify crops for which the amount of research is not proportional to the area of land used for their cultivation. as pigeon peas, millet, plantain, cassava, cashew nuts and sorghum. These are all largely cultivated in the same tropical and subtropical regions where they constitute a major part of the local diet: for example, sorghum in West Africa, millet in West Africa and Southeast Asia, and cassava throughout Africa and Southeast Asia the same areas where NTDs are most prevalent.

However, it cannot be said that there is a complete lack of high-quality research on these crops. Sorghum has had its genome sequenced and published (Nature 457, 551-556; 2009), with three further sequences appearing a couple of years later (Genome Biol. 12, R114; 2011). A reference genome for foxtail millet (Setaria italica) has also been published (Nature Biotechnol. 30, 555-561; 2012), and last year so were draft genomes of domesticated and wild cassava (Nature Commun. 5, 5110; 2014). Such detailed genetic analyses should provide a framework on which more translational studies can build.

The diseases that reduce the harvests of these crops are also a focus of research. For example, a study was published earlier this year about receptors involved in triggering germination in
The most dramatic deviations are in a positive direction: plants that are the subject of far more research than their crop production would suggest. This may be because their use in research is unrelated to their status as a crop. Tobacco and tomato, for example, are the subject of considerable research - they are the fifth and sixth most researched crop plants, respectively because they are commonly used models, particularly for disease resistance and development. Fruit is also disproportionately researched considering the relatively small size of their harvest, as are crops that could be considered luxuries, such as tea, coffee, cocoa and asparagus - 'cash crops', rather than staples.

However, there are also substantially under-researched or neglected crops, such
Striga hermonthica (Science 349, 864-868; 2015), a destructive parasitic plant that preys on sorghum and other grain crops across Africa and beyond. There has also been a recent project by Queen's University Belfast (funded by the Bill \& Melinda Gates Foundation) to develop safe and effective treatments against nematode species affecting sub-Saharan crops such as plantain.

It is a cruel, if unsurprising, irony that the communities that suffer most from NTDs are sustained by crops that are themselves relatively neglected by science. Poverty is a global problem, but its solutions are overwhelmingly local - whether it be the provision of clean water and sanitation, or providing communities with highyielding resilient crops suited to their local environment. 